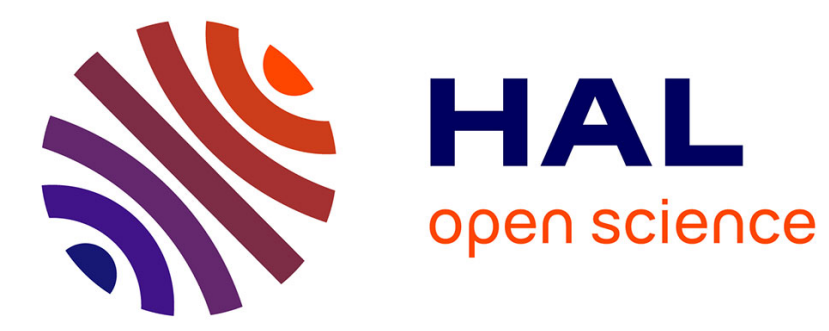

\title{
Robust stability analysis and implementation of Persidskii systems
}

Denis Efimov, A Yu Aleksandrov

\section{To cite this version:}

Denis Efimov, A Yu Aleksandrov. Robust stability analysis and implementation of Persidskii systems.

Proc. IEEE CDC, Dec 2019, Nice, France. hal-02418534

\section{HAL Id: hal-02418534 \\ https://hal.inria.fr/hal-02418534}

Submitted on 18 Dec 2019

HAL is a multi-disciplinary open access archive for the deposit and dissemination of scientific research documents, whether they are published or not. The documents may come from teaching and research institutions in France or abroad, or from public or private research centers.
L'archive ouverte pluridisciplinaire HAL, est destinée au dépôt et à la diffusion de documents scientifiques de niveau recherche, publiés ou non, émanant des établissements d'enseignement et de recherche français ou étrangers, des laboratoires publics ou privés. 


\title{
Robust stability analysis and implementation of Persidskii systems
}

\author{
D. Efimov, A. Yu. Aleksandrov
}

\begin{abstract}
A class of generalized nonlinear Persidskii systems is considered in the paper. The conditions of input-to-state and integral input-to-state stability are established, which can be checked using linear matrix inequalities. The issues of discretization of this class of dynamics are analyzed using the Euler methods. The proposed theory is applied to a Lotka-Volterra model.
\end{abstract}

Analysis of stability of nonlinear dynamical systems is a complex problem, that is why there are various notions and concepts used in the theory of control and systems [1]. The main approach for verification of stability in the nonlinear framework is based on the Lyapunov function method [2]-[4] and its extensions [5]-[8]. The drawback of this approach is that there is no technique assigning a corresponding Lyapunov function to a generic nonlinear system. There are solutions for different canonical forms of nonlinear dynamics: Lurie systems, Lipschitz dynamics, homogeneous systems, Persidskii systems, etc.

In this note the attention is focused on a class of Persidskii systems, which have been first studied in [9] with the Lyapunov functions being a linear combination of the integrals of the nonlinearities, and next by Persidskii in [10], where he extended the previous work by introducing in Lyapunov function a combination of the absolute values of the states. This class of systems has been widely investigated in the context of diagonal stability [11], neural networks [12]-[14] and in other applications [15]. In this paper we are going to further extend these results.

The stability properties, which are studied in this work, are formulated in the framework of input-to-state stable systems [16]. These concepts allow the robust stability of nonlinear systems to be assessed with respect to external inputs, which is important and appreciated in the applications, where presence of different sources of uncertainty is inevitable.

The principal goal in this work is to introduce a class of generalized Persidskii systems and to analyze their robust stability. To this end a special structure of Lyapunov function is proposed, and it is shown that its properties, as well as for its derivative, can be investigated by solving linear matrix inequalities (LMIs). Next, it is demonstrated that for discretization of this class of nonlinear dynamical systems, the Euler method has an advantage to preserve the stability of trajectories under mild restrictions (for nonlinear systems it is not always the case [17], [18]). Finally, a

D. Efimov is with Inria, Univ. Lille, CNRS, UMR 9189 - CRIStAL, F59000 Lille, France and ITMO University, 49 av. Kronverkskiy, 197101 Saint Petersburg, Russia.

A. Yu. Aleksandrov is with ITMO University, 49 av. Kronverkskiy, 197101 Saint Petersburg, Russia.

This work is partially supported by ANR 18 CE40-0008 (Project DIGITSLID), by the Government of Russian Federation (Grant 08-08), by the Ministry of Science and Higher Education of Russian Federation (passport of goszadanie no. 2019-0898) and by the Russian Foundation for Basic Research (grant no. 19-01-00146-a). The results on analysis of Lotka-Volterra systems presented in Section V were developed under support of RSF (grant 17-19-01422) in ITMO University.
Lotka-Volterra system is considered as an example of application of the proposed theory demonstrating its efficiency.

The outline of this paper is as follows. Preliminary results are introduced in Section I. The problem statement is given in Section II. Stability conditions are established in Section III. The implementation issues are studied in Section IV. Simple illustrating application is considered in Section V.

Notation

- $\mathbb{R}_{+}=\{x \in \mathbb{R}: x \geq 0\}$, where $\mathbb{R}$ is the set of real number.

- $|\cdot|$ denotes the absolute value in $\mathbb{R},\|\cdot\|$ denotes the Euclidean norm on $\mathbb{R}^{n}$.

- For a (Lebesgue) measurable function $d: \mathbb{R}_{+} \rightarrow \mathbb{R}^{m}$ define the norm $\|d\|_{\left[t_{0}, t_{1}\right)}=\operatorname{ess} \sup _{t \in\left[t_{0}, t_{1}\right)}\|d(t)\|$, then $\|d\|_{\infty}=$ $\|d\|_{[0,+\infty)}$ and the set of $d(t)$ with the property $\|d\|_{\infty}<$ $+\infty$ we further denote as $\mathcal{L}_{\infty}^{m}$ (the set of essentially bounded measurable functions).

- A continuous function $\alpha: \mathbb{R}_{+} \rightarrow \mathbb{R}_{+}$belongs to the class $\mathcal{K}$ if $\alpha(0)=0$ and the function is strictly increasing. The function $\alpha: \mathbb{R}_{+} \rightarrow \mathbb{R}_{+}$belongs to the class $\mathcal{K}_{\infty}$ if $\alpha \in \mathcal{K}$ and it is increasing to infinity. A continuous function $\beta: \mathbb{R}_{+} \times \mathbb{R}_{+} \rightarrow$ $\mathbb{R}_{+}$belongs to the class $\mathcal{K} \mathcal{L}$ if $\beta(\cdot, t) \in \mathcal{K}_{\infty}$ for each fixed $t \in \mathbb{R}_{+}$and $\lim _{t \rightarrow+\infty} \beta(s, t)=0$ for each fixed $s \in \mathbb{R}_{+}$.

- The notation $D V(x) f(x)$ stands for the directional derivative of a continuously differentiable function $V$ with respect to the vector field $f$ evaluated at point $x$.

- Denote the identity matrix of dimension $n \times n$ by $I_{n}$, the vector of dimension $n$ with all elements equal 1 by $1_{n}$, and $\operatorname{diag}\{g\}$ represents a diagonal matrix of dimension $n \times n$ with a vector $g \in \mathbb{R}^{n}$ on the main diagonal.

- A series of integers $1,2, \ldots, n$ is denoted by $\overline{1, n}$.

\section{Preliminaries}

Consider a nonlinear system:

$$
\dot{x}(t)=f(x(t), d(t)), t \geq 0,
$$

where $x(t) \in \mathbb{R}^{n}$ is the state, $d(t) \in \mathbb{R}^{m}$ is the external input, $d \in \mathcal{L}_{\infty}^{m}$, and $f: \mathbb{R}^{n+m} \rightarrow \mathbb{R}^{n}$ is a locally Lipschitz (or Hölder) continuous function, $f(0,0)=0$. In some cases the system (1) is equipped with an output $y \in \mathbb{R}^{p}$ :

$$
y=h(x),
$$

where $h: \mathbb{R}^{n} \rightarrow \mathbb{R}^{p}$ is a continuous function. For an initial condition $x_{0} \in \mathbb{R}^{n}$ and input $d \in \mathcal{L}_{\infty}^{m}$, define the corresponding solutions by $x\left(t, x_{0}, d\right)$ for any $t \geq 0$ for which the solution exists.

In this work we will be interested in the following stability properties [19]:

Definition 1. The system (1) is called input-to-state practically stable (ISpS), if for any input $d \in \mathcal{L}_{\infty}^{m}$ and any $x_{0} \in \mathbb{R}^{n}$ there are some functions $\beta \in \mathcal{K} \mathcal{L}, \gamma \in \mathcal{K}$ and a constant $c \geq 0$ such that

$$
\left\|x\left(t, x_{0}, d\right)\right\| \leq \beta\left(\left\|x_{0}\right\|, t\right)+\gamma\left(\|d\|_{[0, t)}\right)+c \quad \forall t \geq 0 .
$$


The function $\gamma$ is called nonlinear asymptotic gain. The system is called input-to-state stable (ISS) if $c=0$.

Definition 2. The system (1) is called iISS, if there are some functions $\alpha \in \mathcal{K}_{\infty}, \gamma \in \mathcal{K}$ and $\beta \in \mathcal{K} \mathcal{L}$ such that for any $x_{0} \in \mathbb{R}^{n}$ and $d \in \mathcal{L}_{\infty}^{m}$ the estimate holds:

$$
\alpha\left(\left\|x\left(t, x_{0}, d\right)\right\|\right) \leq \beta\left(\left\|x_{0}\right\|, t\right)+\int_{0}^{t} \gamma(\|d(s)\|) d s \quad \forall t \geq 0 .
$$

Definition 3. The system (1), (2) with $d=0$ is called Output-toState Stable (OSS), if there are functions $\beta \in \mathcal{K} \mathcal{L}$ and $\gamma \in \mathcal{K}$ such that for all $x_{0} \in \mathbb{R}^{n}$

$$
\left\|x\left(t, x_{0}, 0\right)\right\| \leq \beta\left(\left\|x_{0}\right\|, t\right)+\gamma\left(\|y\|_{[0, t)}\right) \quad \forall t \in\left[0, t_{\max }\left(x_{0}\right)\right) .
$$

In this definition $t_{\max }\left(x_{0}\right) \leq+\infty$ determines the interval of solutions existence for the system (1), (2); $y(t)=h\left[x\left(t, x_{0}, 0\right)\right]$ is the output solution.

Definition 4. The system (1), (2) is called input-output-to-state stable (IOSS) if there are functions $\beta \in \mathcal{K} \mathcal{L}$ and $\gamma_{1}, \gamma_{2} \in \mathcal{K}$ such that for all $x_{0} \in \mathbb{R}^{n}, d \in \mathcal{L}_{\infty}^{m}$ and all $t \in\left[0, t_{\max }\left(x_{0}, d\right)\right)$,

$$
\left\|x\left(t, x_{0}, d\right)\right\| \leq \beta\left(\left\|x_{0}\right\|, t\right)+\gamma_{1}\left(\|d\|_{[0, t)}\right)+\gamma_{2}\left(\|y\|_{[0, t)}\right) .
$$

Where again $t_{\max }\left(x_{0}, d\right) \leq+\infty$ defines the interval of existence of the system (1), (2) solutions, $y(t)=h\left[x\left(t, x_{0}, d\right)\right]$ is the corresponding output trajectory.

These properties have the following Lyapunov function characterizations:

Definition 5. A smooth function $V: \mathbb{R}^{n} \rightarrow \mathbb{R}_{+}$is called ISpS Lyapunov function for the system (1) if for all $x \in \mathbb{R}^{n}, d \in \mathbb{R}^{m}$ and some $r \geq 0, \alpha_{1}, \alpha_{2}, \alpha_{3} \in \mathcal{K}_{\infty}$ and $\theta \in \mathcal{K}$ :

$$
\begin{aligned}
\alpha_{1}(\|x\|) & \leq V(x) \leq \alpha_{2}(\|x\|), \\
D V(x) f(x, d) & \leq r+\theta(\|d\|)-\alpha_{3}(\|x\|) .
\end{aligned}
$$

Such a function $V$ is called ISS Lyapunov function if $r=0$, and it is iISS Lyapunov function if instead $\alpha_{3}: \mathbb{R}_{+} \rightarrow \mathbb{R}_{+}$is a positive definite function.

Note that an ISS Lyapunov function can also satisfy the following equivalent condition for some $\chi \in \mathcal{K}$ :

$$
\|x\|>\chi(\|d\|) \Rightarrow D V(x) f(x, d) \leq-\alpha_{3}(\|x\|) .
$$

Definition 6. A smooth function $V: \mathbb{R}^{n} \rightarrow \mathbb{R}_{+}$is called OSS Lyapunov function for the system (1), (2) with $d=0$ if for all $x \in \mathbb{R}^{n}$ there are functions $\alpha_{1}, \alpha_{2}, \alpha_{3} \in \mathcal{K}_{\infty}, \sigma \in \mathcal{K}$ such that:

$$
\begin{gathered}
\alpha_{1}(\|x\|) \leq V(x) \leq \alpha_{2}(\|x\|), \\
D V(x) f(x, 0) \leq-\alpha_{3}(\|x\|)+\sigma(\|y\|) .
\end{gathered}
$$

Definition 7. A smooth function $V$ is called IOSS Lyapunov function for the system (1), (2) if for all $x \in \mathbb{R}^{n}, d \in \mathbb{R}^{m}$ and some functions $\alpha_{1}, \alpha_{2}, \alpha_{3} \in \mathcal{K}_{\infty}, \sigma_{1}, \sigma_{2} \in \mathcal{K}$ the inequalities hold:

$$
\begin{gathered}
\alpha_{1}(\|x\|) \leq V(x) \leq \alpha_{2}(\|x\|), \\
D V(x) f(x, d) \leq-\alpha_{3}(\|x\|)+\sigma_{1}(\|d\|)+\sigma_{2}(\|y\|) .
\end{gathered}
$$

Theorem 1. The system (1) is ISS (ISpS, iISS) iff it admits an ISS (ISpS, iISS) Lyapunov function.

Theorem 2. The system (1), (2) is IOSS (OSS for $d=0$ ) iff it admits an IOSS (OSS) Lyapunov function.

Note that, if the system (1) is ISS, then it is also iISS.

\section{Problem Statement}

Consider the following class of extended Persidskii systems:

$$
\begin{aligned}
& \dot{x}(t)=A_{0} x(t)+\sum_{j=1}^{M} A_{j} f^{j}(x(t))+d(t), t \geq 0, \\
& y(t)=h(x(t))=\left[\begin{array}{c}
C_{0} x(t) \\
C_{1} f^{1}(x(t)) \\
\vdots \\
C_{M} f^{M}(x(t))
\end{array}\right],
\end{aligned}
$$

where $x(t)=\left[x_{1}(t) \ldots x_{n}(t)\right]^{\top} \in \mathbb{R}^{n}$ is the state vector, $x(0) \in$ $\mathbb{R}^{n} ; y(t) \in \mathbb{R}^{p}$ is the output signal, $p=\sum_{k=0}^{M} p_{k}$ and $C_{k} \in \mathbb{R}^{p_{k} \times n}$ for $k=\overline{0, M}$ (some $p_{k}$ can be zero that implies independence of the output on $f^{k}(x)$ ); and $d(t) \in \mathbb{R}^{n}$ is the external perturbation, $d \in$ $\mathcal{L}_{\infty}^{n} ; f^{j}(x)=\left[f_{1}^{j}\left(x_{1}\right) \ldots f_{n}^{j}\left(x_{n}\right)\right]^{\top}, j=\overline{1, M}$ are the functions ensuring existence of solutions of the system (3) in the forward time at least locally, the matrices $A_{k} \in \mathbb{R}^{n \times n}$ for $k=\overline{0, M}$.

Assumption 1. For any $i=\overline{1, n}, j=\overline{1, M}$ :

$$
s f_{i}^{j}(s)>0 \quad \forall s \in \mathbb{R} \backslash\{0\} ;
$$

there exists $m \in\{0, \ldots, M\}$ such that for all $i=\overline{1, n}, z=\overline{1, m}$ :

$$
\lim _{s \rightarrow \pm \infty} f_{i}^{z}(s)= \pm \infty
$$

there exists $\mu \in\{m, \ldots, M\}$ such that for all $i=\overline{1, n}, z=\overline{1, \mu}$ :

$$
\lim _{s \rightarrow \pm \infty} \int_{0}^{s} f_{i}^{z}(\sigma) d \sigma=+\infty .
$$

In the assumption above, first, it is stated that all nonlinearities belong to a sector and may take zero values at zero only. Second, it is supposed that some of the nonlinearities are radially unbounded, and $m=0$ corresponds to the case when all nonlinearities are bounded (at least for negative or positive argument). Finally, it is stated that some of these nonlinearities have unbounded integral, and clearly if $m>0$, then all radially unbounded nonlinearities also have unbounded integrals, thus $\mu \geq m$ due to the introduced sector condition. Such an ordering of $f^{j}$ can be obtained after a proper re-indexing and decomposition of a nonlinear system dynamics, and the principal restriction is the sector condition.

If $A_{r}=0$ for all $r=\overline{0, M-1}$ and $\mu=m=M$, then we recover the system studied by Persidskii in the conventional framework [10].

Our goal is to propose constructive conditions to check for (3) on possessiveness of ISS, iISS, OSS and IOSS properties.

\section{STABILITY CONDITIONS}

In this section, for two diagonal matrices $\Lambda^{1}, \Lambda^{2} \in \mathbb{R}^{n \times n}$, their maximum $\max _{z=1,2} \Lambda^{z}$ is understood element-wise. The main result of this paper is as follows:

Theorem 3. Let Assumption 1 be satisfied and there exist $P=$ $P^{\top} \in \mathbb{R}^{n \times n} ; \Xi^{k}=\operatorname{diag}\left\{\xi^{k}\right\}$ with $\xi^{k}=\left[\xi_{1}^{k} \ldots \xi_{n}^{k}\right]^{\top} \in \mathbb{R}^{n}$ for $k=\overline{0, M} ; \Lambda^{j}=\operatorname{diag}\left\{\lambda^{j}\right\}$ with $\lambda^{j}=\left[\lambda_{1}^{j} \ldots \lambda_{n}^{j}\right]^{\top} \in \mathbb{R}^{n}$ for $j=\overline{1, M} ; \Upsilon_{s, j}=\operatorname{diag}\left\{v^{s, j}\right\}$ with $v^{s, j}=\left[v_{1}^{s, j} \ldots v_{n}^{s, j}\right]^{\top} \in \mathbb{R}^{n}$ 
for $s=\overline{0, M}$ and $j=\overline{s+1, M}, \Gamma=\Gamma^{\top}>0$ and $\chi \geq 0$ such that

$$
\begin{gathered}
\Lambda^{j} \geq 0, j=\overline{1, M} ; \quad \Xi^{k} \geq 0, k=\overline{0, M} \\
P>0 \text { or } P \geq 0, \max _{z=\overline{1, \mu}} \Lambda^{z}>0 ; \\
\Upsilon_{s, j} \geq 0, s=\overline{0, M}, j=\overline{s+1, M} \\
\sum_{k=0}^{M} \Xi^{k}+2 \sum_{s=0}^{M} \sum_{j=s+1}^{M} \Upsilon_{s, j}>0 ; Q \leq 0
\end{gathered}
$$

where

$$
\begin{gathered}
Q=\left[\begin{array}{cccccc}
Q_{1,1} & Q_{1,2} & Q_{1,3} & \cdots & Q_{1, M+1} & P \\
Q_{1,2}^{+} & Q_{2,2} & Q_{2,3} & \cdots & Q_{2, M+1} & \Lambda^{1} \\
Q_{1,3}^{\top} & Q_{2,3}^{\top} & Q_{3,3} & \cdots & Q_{3, M+1} & \Lambda^{2} \\
\vdots & \vdots & \vdots & \ddots & \vdots & \vdots \\
Q_{1, M+1}^{\top} & Q_{2, M+1}^{\top} & Q_{3, M+1}^{\top} & \cdots & Q_{M+1, M+1} & \Lambda^{M} \\
P & \Lambda^{1} & \Lambda^{2} & \cdots & \Lambda^{M} & -\Gamma
\end{array}\right] \\
Q_{1,1}=A_{0}^{\top} P+P A_{0}+\Xi^{0}-\chi C_{0}^{\top} C_{0} ; \\
Q_{j+1, j+1}=A_{j}^{\top} \Lambda^{j}+\Lambda^{j} A_{j}+\Xi^{j}-\chi C_{j}^{\top} C_{j}, j=\overline{1, M} ; \\
Q_{1, j+1}=P A_{j}+A_{0}^{\top} \Lambda^{j}+\Upsilon_{0, j}, j=\overline{1, M} ; \\
Q_{s+1, j+1}=A_{s}^{\top} \Lambda^{j}+\Lambda^{s} A_{j}+\Upsilon_{s, j}, s=\overline{1, M}, j=\overline{s+1, M} .
\end{gathered}
$$

Then the system (3) is

- iISS for $\chi=0$;

- ISS provided that $m \geq 1, \chi=0$ and the last condition in (4) is strengthened to

$$
\sum_{k=0}^{m} \Xi^{k}+2 \sum_{s=0}^{m} \sum_{j=s+1}^{m} \Upsilon_{s, j}>0
$$

- IOSS if the conditions of ISS are verified with some $\chi>0$.

All proofs are skipped due to space limitations. The proof of this theorem is based on analysis of Lyapunov function

$$
V(x)=x^{\top} P x+2 \sum_{j=1}^{M} \sum_{i=1}^{n} \lambda_{i}^{j} \int_{0}^{x_{i}} f_{i}^{j}(s) d s,
$$

whose desired properties follow the introduced hypotheses and (4).

In this result, the matrices $P$ and $\Lambda^{j}$ for $j=\overline{1, M}$ serve as solutions of the Lyapunov equation for the matrices $A_{0}$ and $A_{j}$ for $j=\overline{1, M}$, respectively. The matrices $\Xi^{k}$ for $k=\overline{0, M}$ are used in $Q$ in order to relax the stability requirements for each matrix $A_{k}$ for $k=\overline{0, M}$ by looking for their total influence on the system performance (i.e. the condition $\sum_{k=0}^{M} \Xi^{k}>0$ ). The matrices $\Upsilon_{s, j}$ for $s=\overline{0, M}$ and $j=\overline{s+1, M}$ are introduced in order to reduce the conservatism of the condition $Q \leq 0$, since all crossterms of the form $x_{i} f_{i}^{j}\left(x_{i}\right)$ or $f_{i}^{j}\left(x_{i}\right) f_{i}^{s}\left(x_{i}\right)$ with $i=\overline{1, n}$, which appear in the off-diagonal blocks of $Q$, should not be considered as "perturbations" in verification of the restriction $Q \leq 0$ provided that they have a non-positive multiplier due to the sector properties of the functions $f_{i}^{j}$.

Remark 1. We can also relax the conditions on positiveness of the matrices $\Lambda^{z}$ for $z=\overline{1, \mu}$ if we assume, for example, that there exist $\kappa_{z}>0$ such that

$$
\left\|f^{z}(x)\right\|^{2} \geq \kappa_{z}\|x\|^{2}
$$

for all $x \in \mathbb{R}^{n}$ and all $z=\overline{1, \mu}$, then the corresponding LMIs in (4) can be replaced with the following ones:

$$
\begin{gathered}
\Lambda^{j} \geq 0, j=\overline{\mu+1, M} ; \quad \Xi^{k} \geq 0, k=\overline{0, M} ; \\
P+\sum_{z=1}^{\mu} \kappa_{z} \min _{i=\overline{1, n}} \lambda_{i}^{z} I_{n}>0 ; \\
\Upsilon_{s, j} \geq 0, s=\overline{0, M}, j=\overline{s+1, M} ; \\
Q \leq 0 ; \Theta \geq 0 \\
\Theta+\sum_{k=\mu+1}^{M} \Xi^{k}+2 \sum_{s=0}^{M} \sum_{j=s+1}^{M} \Upsilon_{s, j}>0,
\end{gathered}
$$

where $\Theta=\Xi^{0}+\sum_{z=1}^{\mu} \kappa_{z} \min _{i=\overline{1, n}} \xi_{i}^{z} I_{n}$. Of course, similar relaxations can also be imposed if one nonlinearity has a higher amplitude than another, e.g.

$$
\left\|f^{z_{1}}(x)\right\|^{2} \geq \kappa_{z_{1}, z_{2}}\left\|f^{z_{2}}(x)\right\|^{2}
$$

for some $z_{1} \neq z_{2} \in\{1, \ldots, M\}$ and some $\kappa_{z_{1}, z_{2}}>0$ (the same for the cross terms $x^{\top} \Upsilon_{0, j} f^{j}(x)$ with $\left.j=\overline{1, M}\right)$.

Remark 2. In the ISS case, for $j=\overline{\mu+1, M}$ the sector requirement on $f_{i}^{j}$ introduced in Assumption 1 can be relaxed as follows:

$$
s f_{i}^{j}(s) \geq 0 \quad \forall s \in \mathbb{R}
$$

with $i=\overline{1, n}$. We can also extend such a consideration for all $j=\overline{1, M}$, but then we have to carefully analyze the issues with the absence of additional equilibria, or consider the multistability case (bi-stability, presence of limit cycles or other oscillating modes).

Remark 3. It is straightforward to conclude that in order to satisfy the condition $Q \leq 0$ the matrices $A_{j}^{\top} \Lambda^{j}+\Lambda^{j} A_{j}$, which appear on the main diagonal of $Q$ for $j=\overline{1, M}$, have to be nonnegative definite also. Recall that $\Lambda^{j}$ are diagonal matrices, then for a stable Metzler matrix $A_{j}$ (a matrix with all nonnegative elements outside of the main diagonal) existence of such a diagonal matrix $\Lambda^{j}$ is necessary and sufficient for the stability. Note also that if in the system (3) the matrices $A_{k}$ for $k=\overline{0, M}$, are Metzler, then the corresponding terms $\sum_{i=1}^{n} \lambda_{i}^{j} \int_{0}^{x_{i}} f_{i}^{j}(s) d s$ in the Lyapunov function can be replaced by $\sum_{i=1}^{n} \lambda_{i}^{j}\left|x_{i}\right|$ [10], and the Lyapunov function can be simplified to the form:

$$
V(x)=\sum_{i=1}^{n} \lambda_{i}\left|x_{i}\right|
$$

with $P=0$, whose derivative can be rewritten as follows (denote $\operatorname{sign}(x)=\left[\operatorname{sign}\left(x_{1}\right) \ldots \operatorname{sign}\left(x_{n}\right)\right]^{\top}$ and $\Lambda=\operatorname{diag}\{\lambda\}$ with $\lambda=$ $\left.\left[\lambda_{1} \ldots \lambda_{n}\right]^{\top} \in \mathbb{R}^{n}\right)$ :

$$
\begin{aligned}
\dot{V} & =\dot{x}^{\top} \Lambda \operatorname{sign}(x) \\
& =\left(A_{0} x+\sum_{j=1}^{M} A_{j} f^{j}(x)+d\right)^{\top} \Lambda \operatorname{sign}(x) .
\end{aligned}
$$

Thus, if the following linear programming problem with respect to $\lambda$ and $\xi^{k}=\left[\xi_{1}^{k} \ldots \xi_{n}^{k}\right]^{\top} \in \mathbb{R}^{n}, k=\overline{0, M}$ is satisfied:

$$
A_{k}^{\top} \lambda=-\xi^{k} \leq 0, k=\overline{0, M} ; \quad \sum_{k=0}^{M} \xi^{k}>0,
$$

then

$$
\dot{V} \leq-\sum_{i=1}^{n} \xi_{i}^{0}\left|x_{i}\right|-\sum_{j=1}^{M} \sum_{i=1}^{n} \xi_{i}^{j}\left|f_{i}^{j}\left(x_{i}\right)\right|+\sum_{i=1}^{n} \lambda_{i}\left|d_{i}\right|,
$$


and the same conclusions can be deduced. The shortage of this approach is that in such a case all matrices $A_{k}$ for $k=\overline{0, M}$ have to possess a common Lyapunov function.

The statement of Theorem 3 and the remarks above describe the basic ideas of the approach and various relaxations or auxiliary results that can be obtained around (these developments are left for future research). Let us also formulate the OSS case, which can also be useful for stability analysis:

Corollary 1. Let all conditions of Theorem 3 be satisfied for $\Gamma=0$ and

$$
Q=\left[\begin{array}{ccc}
Q_{1,1} & \cdots & Q_{1, M+1} \\
\vdots & \ddots & \vdots \\
Q_{1, M+1}^{\top} & \cdots & Q_{M+1, M+1}
\end{array}\right]
$$

where the elements of the matrix $Q$ are given in Theorem 3. Then the system (3) with $d(t)=0$ for all $t \geq 0$ is

- globally asymptotically stable with $\chi=0$;

- OSS provided that $m \geq 1, \chi>0$ and the last condition in (4) is strengthened to

$$
\sum_{k=0}^{m} \Xi^{k}+2 \sum_{s=0}^{m} \sum_{j=s+1}^{m} \Upsilon_{s, j}>0
$$

\section{IMPLEMENTATION WITH THE USE OF EULER METHOD}

For the conventional Persidskii system, the issues of application for discretization of solutions of the explicit Euler method have been analyzed in [20], [21], and these results have been developed to the Persidskii systems with delays in [22]. Application of the explicit and the implicit Euler methods to the extended class of Persidskii systems (3) with $d(t)=0$ for all $t \geq 0$ is considered in this section.

Denote by

$$
F(x)=A_{0} x+\sum_{j=1}^{M} A_{j} f^{j}(x)
$$

the right-hand side of the system (3) in this case, and assume that the system (3) admits the zero solution to be asymptotically stable. We will look for conditions of the preservation of the asymptotic stability after discretization of (3) using the Lyapunov function $V(x)$ constructed in the proof of Theorem 3.

First, consider an application of the explicit Euler method:

$$
z(k+1)=z(k)+h F(z(k)), z(0)=x_{0} \in \mathbb{R}^{n},
$$

where $z(k) \in \mathbb{R}^{n}$ is an estimate of the solution $x\left(h k, x_{0}, 0\right)$ of the system (3), $h$ is a digitization step and $k=0,1, \ldots$ is the iteration number.

Theorem 4. Let the functions $f^{j}(x), j=\overline{1, M}$ be locally Lipschitz continuous. If the conditions of Corollary 1 are fulfilled with $\chi=0$ and $\Xi^{0}>0$, then for any $H>0$ there exists a constant $h_{0}>0$ such that the zero solution of (5) is asymptotically stable for all $h \in\left(0, h_{0}\right)$ and all initial conditions $x_{0} \in \Omega_{H}=\left\{x \in \mathbb{R}^{n}\right.$ : $V(x)<H\}$.

Next, consider an application of the implicit Euler method:

$$
z(k+1)=z(k)+h F(z(k+1)), z(0)=x_{0} \in \mathbb{R}^{n} .
$$

Theorem 5. Let the functions $f^{j}(x)$ be continuously differentiable and $\frac{\partial f^{j}(x)}{\partial x} \geq 0$ for all $x \in \mathbb{R}^{n}, j=\overline{1, M}$. If the conditions of Corollary 1 are fulfilled with $\chi=0$, then for any $H>0$ there exists a constant $h_{0}>0$ such that for all $h \in\left(0, h_{0}\right)$ and all initial conditions $x_{0} \in \Omega_{H}$ :

(i) the solution $z(k)$ of (6) is defined for all $k=0,1, \ldots$;

(ii) the zero solution of (6) is asymptotically stable.

Comparing the results of theorems 4 and 5 it is worth to stress that the limit $h_{0}$ on the admissible discretization step $h$ for the implicit method comes form the conditions of existence of $z(k+1)$ in (6) (this scheme is always stable under the hypotheses of Theorem 5), while for the explicit method (5) the discretization may loose its stability if the step $h$ is not sufficiently small. Theorem 5 also imposes additional conditions on monotonicity of the nonlinearities (i.e. $\frac{\partial f^{j}(x)}{\partial x} \geq 0$ for all $x \in \mathbb{R}^{n}$ ), but under less restrictive stability conditions (the requirement $\Xi^{0}>0$ is not introduced).

\section{ANALYSIS OF A MODIFIED GENERALIZED LOTKA-VOLTERRA DYNAMICS}

The generalized Lotka-Volterra equations are frequently utilized to investigate competition and trophic relationships between an arbitrary number of species [23] (in biology or finance, for example). However, this model does not possess some important biological features (e.g. predator preference and nonlinear functional responses), that is why several its modifications have been proposed, and among them the following one with a support of mutualistic interactions [24], [25]:

$$
\dot{x}(t)=\operatorname{diag}\{x(t)\}\left(b+A x(t)+A_{2} \varphi(x(t))\right), t \geq 0,
$$

where $x(t) \in \mathbb{R}_{+}^{n}$ is the vector of the populations of $n$ biological species, $x(0) \in \mathbb{R}_{+}^{n} ; b \in \mathbb{R}^{n}$ corresponds to the intrinsic birth or death rates of the species, $A \in \mathbb{R}^{n \times n}$ is the community matrix, and $A_{2} \in \mathbb{R}^{n \times n}$ is the mutualistic interaction strength between the species; $\varphi(x(t))=\left[\varphi_{1}\left(x_{1}\right) \ldots \varphi_{n}\left(x_{n}\right)\right]^{\top}=\left[\frac{x_{1}}{r_{1}+x_{1}} \ldots \frac{x_{n}}{r_{n}+x_{n}}\right]^{\top}$ is the vector of Michaelis-Menten functions with $r=\left[r_{1} \ldots r_{n}\right]^{+} \in$ $\mathbb{R}^{n}$ being the half-saturation constants.

Assume that there exists a unique equilibrium point $x^{e q} \in \mathbb{R}_{+}^{n}$, with $x_{i}^{e q}>0$ for all $i=\overline{1, n}$, that is the solution of the equation

$$
b+A x^{e q}+A_{2} \varphi\left(x^{e q}\right)=0,
$$

and consider a change of coordinates:

$$
\epsilon=\ln (x)-\ln \left(x^{e q}\right), x=\operatorname{diag}\left\{x^{e q}\right\} e^{\epsilon},
$$

where the operations $\ln (\cdot)$ and its inverse $e^{(\cdot)}$ are understood element-wise. The variable $\epsilon(t)$ measures a nonlinear deviation of $x(t)$ with respect to the steady state $x^{e q}$ and whose dynamics take the form:

$$
\begin{aligned}
\dot{\epsilon}(t)= & A \operatorname{diag}\left\{x^{e q}\right\}\left[e^{\epsilon(t)}-1_{n}\right] \\
& +A_{2}\left[\varphi\left(\operatorname{diag}\left\{x^{e q}\right\} e^{\epsilon(t)}\right)-\varphi\left(x^{e q}\right)\right] \\
= & A_{1} f^{1}(\epsilon(t))+A_{2} f^{2}(\epsilon(t)),
\end{aligned}
$$

where

$$
\begin{gathered}
A_{1}=A \operatorname{diag}\left\{x^{e q}\right\}, f^{1}(\epsilon)=e^{\epsilon}-1_{n}, \\
f^{2}(\epsilon)=\varphi\left(\operatorname{diag}\left\{x^{e q}\right\} e^{\epsilon}\right)-\varphi\left(x^{e q}\right) .
\end{gathered}
$$




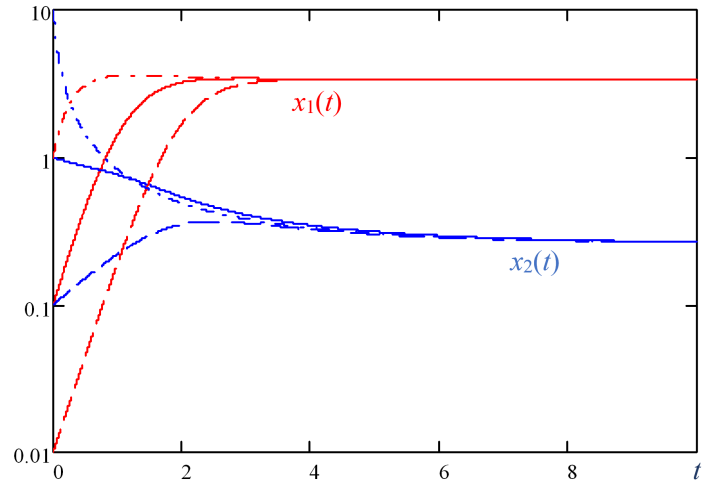

Figure 1. The results of simulation for Lotka-Volterra model

Assumption 1 is satisfied with $m=0$ and $\mu=2$, then the results of Theorem 3 or Corollary 1 can be applied, and the system admits a Lyapunov function:

$$
\begin{aligned}
V(x)= & \sum_{i=1}^{n} \lambda_{i}^{1}\left(\frac{x_{i}}{x_{i}^{e q}}-\ln \left(\frac{x_{i}}{x_{i}^{e q}}\right)-1\right) \\
& +\sum_{i=1}^{n} \lambda_{i}^{2}\left(\ln \left(\frac{r_{i}+x_{i}}{r_{i}+x_{i}^{e q}}\right)-\frac{x_{i}^{e q} \ln \left(\frac{x_{i}}{x_{i}^{e q}}\right)}{r_{i}+x_{i}^{e q}}\right),
\end{aligned}
$$

where $\lambda^{1}$ and $\lambda^{2}$ are solutions of (4) with the matrix $Q$ given in Corollary 1 .

\section{Example 1. Let}

$$
A=\left[\begin{array}{cc}
-0.7 & 0.5 \\
-0.2 & -1.5
\end{array}\right], A_{2}=\left[\begin{array}{cc}
-1 & -0.2 \\
0 & 0.5
\end{array}\right], b=\left[\begin{array}{l}
3 \\
1
\end{array}\right]
$$

then the conditions of Corollary 1 for asymptotic stability are verified, and the system trajectories for three different initial conditions are shown in Fig. 1 (the method (5) has been applied with $h=0.001$ ).

Other possible applications in the domain of population dynamics and systems biology can be related with different dynamical systems based on mass-balance equations, e.g. circadian oscillations in [26] or chemostat [27]. An application of the proposed approach for analysis of stability of an interval predictor is given in [28].

\section{CONCLUSIONS}

The framework of Persidskii systems is revisited and new conditions of ISS, iISS, IOSS and OSS are proposed, which are formulated in terms of LMIs. The proposed theory is applied for stability analysis of Euler discretization and of a population dynamics. Several directions of future research are indicated, design of a finite-time or a fixed-time stabilizing forwarding control is one of them.

\section{REFERENCES}

[1] H. K. Khalil, Nonlinear Systems, ser. NJ 07458. Upper Saddle River: Prentice-Hall, 1996.

[2] A. M. Lyapunov, "Stability of motion: General problem," Internat. J. Control, vol. 55, no. 3, pp. 520-790, march 1992, lyapunov Centenary issue.

[3] I. Malkin, Theory of Stability of Motion. US Atomic Energy Commission, 1952, vol. Translation Series: Physics and Mathematics.

[4] W. Hahn, Stability of Motion. New York: Springer-Verlag Berlin Heidelberg, 1967.
[5] J. LaSalle and S. Lefchetz, Stability by Liapunov's Direct Method with Aplications. Academic Press, New York, 1961.

[6] A. van der Schaft, $L_{2}$-gain and passivity techniques in nonlinear control, ser. Lecture Notes in Control and Information Sciences. London: Springer-Verlag London Ltd., 1996, vol. 218.

[7] Y. Lin, E. D. Sontag, and Y. Wang, "A smooth converse lyapunov theorem for robust stability," SIAM Journal on Control and Optimization, vol. 34, no. 1, pp. 124-160, 1996.

[8] A. Bacciotti and L. Rosier, Lyapunov Functions and Stability in Control Theory, 2nd ed. Springer, Berlin, 2005.

[9] E. Barbashin, "On construction of lyapunov functions for nonlinear systems," in Proc. 1st IFAC World Congress, Moscow, 1961, pp. 742751.

[10] S. Persidskii, "Problem of absolute stability," Automation and Remote Control, vol. 12, pp. 1889-1895, 1969.

[11] E. Kazkurewicz and A. Bhaya, Matrix Diagonal Stability in Systems and Computation. Boston: Birkhauser, 1999.

[12] J. Hopfield and D. Tank, "Computing with neural circuits: a model," Science, vol. 233, pp. 625-633, 1986.

[13] E. Sontag, Dealing with Complexity: a Neural Network Approach. London: Springer-Verlag, 1997, ch. Recurrent neural networks: Some systems-theoretic aspects, pp. 1-12.

[14] L. Ferreira, E. Kaszkurewicz, and A. Bhaya, "Solving systems of linear equations via gradient systems with discontinuous righthand sides: application to LS-SVM," IEEE Transactions on Neural Networks, vol. 16, no. 2, pp. 501-505, 2005.

[15] K. Erickson and A. Michel, "Stability analysis of fixed-point digital filters using computer generated Lyapunov functions - Part I: Direct form and coupled form filters," IEEE Trans. on Circuits and Systems, vol. 32, pp. 113-132, 1985.

[16] E. D. Sontag, "The ISS philosophy as a unifying framework for stability-like behavior," in Nonlinear control in the year 2000, Vol. 2 (Paris), ser. Lecture Notes in Control and Inform. Sci. London: Springer, 2001, vol. 259, pp. 443-467.

[17] D. Efimov, A. Polyakov, A. Levant, and W. Perruquetti, "Realization and discretization of asymptotically stable homogeneous systems," IEEE Transactions on Automatic Control, vol. 62, no. 11, pp. 59625969, 2017.

[18] D. Efimov, A. Polyakov, and A. Aleksandrov, "Discretization of homogeneous systems using euler method with a state-dependent step," Automatica, 2019.

[19] S. Dashkovskiy, D. Efimov, and E. Sontag, "Input to state stability and allied system properties," Automation and Remote Control, vol. 72, no. 8, pp. 1579-1614, 2011.

[20] A. Aleksandrov and A. Zhabko, "Preservation of stability under discretization of systems of ordinary differential equations," Siberian Math. J., vol. 51, no. 3, pp. 383-395, 2010.

[21] A. Aleksandrov, Y. Chen, A. Platonov, and L. Zhang, "Stability analysis and uniform ultimate boundedness control synthesis for a class of nonlinear switched difference systems," J. Differ. Equat. Appl., vol. 18, no. 9, pp. 1545-1561, 2012.

[22] A. Aleksandrov and E. Aleksandrova, "Delay-independent stability conditions for a class of nonlinear difference systems," Journal of the Franklin Institute, vol. 355, pp. 3367-3380, 2018.

[23] R. Arditi and L. Ginzburg, How Species Interact: Altering the Standard View on Trophic Ecology. New York: Oxford University Press, 2012.

[24] J. Holland, D. DeAngelis, and J. Bronstein, "Population dynamics and mutualism: Functional responses of benefits and costs," The American Naturalist, vol. 159, pp. 231-244, 2002.

[25] D. Holland, J.N. amd DeAngelis, "A consumer-resource approach to the density-dependent population dynamics of mutualism," Ecology, vol. 91, pp. 1286-1295, 2010.

[26] A. Goldbeter, "A model for circadian oscillations in the drosophila period protein (per)," Proc. Royal Society of London. Series B: Biological Sciences, vol. 261, no. 1362, 1995.

[27] V. Lemesle and J. L. Gouzé, "A simple unforced oscillatory growth model in the chemostat," Bulletin of Mathematical Biology, vol. 70, no. 2, pp. 344-357, 2008.

[28] E. Leurent, D. Efimov, T. Ra-ssi, and W. Perruquetti, "Interval prediction for continuous-time systems with parametric uncertainties," in Proc. IEEE Conference on Decision and Control (CDC), Nice, 2019. 